\title{
Vegetarian diet and incidence of total, ischemic, and hemorrhagic stroke in $\mathbf{2}$ cohorts in Taiwan
}

Tina H.T. Chiu, PhD, RD, * Huai-Ren Chang, MD, PhD, * Ling-Yi Wang, PhD, Chia-Chen Chang, MS, Ming-Nan Lin, MD, MPH, and Chin-Lon Lin, MD

Neurology ${ }^{\circledR}$ 2020;94:e1112-e1121. doi:10.1212/WNL.0000000000009093

\section{Abstract}

\section{Objective}

To determine how a vegetarian diet affects stroke incidence in 2 prospective cohorts and to explore whether the association is modified by dietary vitamin $\mathrm{B}_{12}$ intake.

\section{Methods}

Participants without stroke in the Tzu Chi Health Study (cohort $1, \mathrm{n}=5,050$, recruited in 2007-2009) and the Tzu Chi Vegetarian Study (cohort 2, $\mathrm{n}=8,302$, recruited in 2005) were followed until the end of 2014. Diet was assessed through food frequency questionnaires in both cohorts at baseline. Stroke events and baseline comorbidities were identified through the National Health Insurance Research Database. A subgroup of 1,528 participants in cohort 1 were assessed for serum homocysteine, vitamin $B_{12}$, and folate. Associations between vegetarian diet and stroke incidences were estimated by Cox regression with age as time scale, adjusted for sex, education, smoking, alcohol, physical activities, body mass index (only in cohort 1), hypertension, diabetes, dyslipidemia, and ischemic heart diseases.

\section{Results}

Vegetarians had lower serum vitamin $\mathrm{B}_{12}$ and higher folate and homocysteine than nonvegetarians. In cohort 1, 54 events occurred in 30,797 person-years follow-up. Vegetarians (vs nonvegetarians) experienced lower risk of ischemic stroke (hazard ratio [HR], 0.26; 95\% confidence interval [CI], 0.08-0.88). In cohort 2, 121 events occurred in 76,797 person-years follow-up. Vegetarians (vs nonvegetarians) experienced lower risk of overall stroke ( $\mathrm{HR}, 0.52$; 95\% CI, 0.33-0.82), ischemic stroke (HR, 0.41; 95\% CI, 0.19-0.88), and hemorrhagic stroke (HR, 034; 95\% CI, 0.12-1.00). Our explorative analysis showed that vitamin $\mathrm{B}_{12}$ intake may modify the association between vegetarian diet and overall stroke $(p$ interaction $=0.046)$.

\section{Conclusion}

Taiwanese vegetarian diet is associated with a lower risk of ischemic and hemorrhagic strokes.

\author{
Correspondence \\ Dr. C.-L. Lin \\ cllinmd@tzuchi.com.tw
}

\section{RELATED ARTICLE \\ Editorial \\ Lower risk of stroke with \\ a vegetarian diet \\ Page 463}

MORE ONLINE

Q CME Course

NPub.org/cmelist

\footnotetext{
*These authors contributed equally to this work.

From the Department of Nutritional Science (T.H.T.C.), Fu Jen Catholic University, New Taipei City; Division of Cardiology, Department of Internal Medicine (H.-R.C.), Epidemiology and Biostatistics Consulting Center, Department of Medical Research (L.-Y.W.), and Department of Pharmacy (L.-Y.W.), Buddhist Tzu Chi General Hospital; School of Medicine (H.R.C., C.-L.L.), Tzu Chi University; Department of Medical Research (C.-C.C.), Buddhist Tzu Chi Medical Foundation, Hualien; Departments of Family Medicine (M.-N.L.) and Internal Medicine (C.-L.L.), Dalin Tzu Chi Hospital, Buddhist Tzu Chi Medical Foundation, Chiayi; and Department of Family Medicine, School of Medicine (M.-N.L.), Tzu Chi University, Hualien, Taiwan.

Go to Neurology.org/N for full disclosures. Funding information and disclosures deemed relevant by the authors, if any, are provided at the end of the article. 


\section{Glossary}

$\mathbf{B M I}=$ body mass index; $\mathbf{B P}=$ blood pressure; $\mathbf{C I}$ = confidence interval; $\mathbf{e G F R}=$ estimated glomerular filtration rate; $\mathbf{F F Q}=$ food frequency questionnaire; HR = hazard ratio; HWDC = Health and Welfare Data Science Center; ICD-9-CM = International Classification of Diseases, 9th Revision, Clinical Modification; LDL = low-density lipoprotein; NHIP = National Health Insurance Program; NHIRD = National Health Insurance Research Database; RDA = recommended dietary allowance; SBP = systolic blood pressure; TMAO = trimethylamine $\mathrm{N}$-oxide.

Stroke is the second most common cause of death globally, accounting for about 1 out of every 19 deaths in the United States, ${ }^{1}$ and is the third leading cause of death in Taiwan. ${ }^{2}$ In Taiwan, ischemic subtype accounts for $74 \%$ of all stroke, followed by intracranial hemorrhage (16.1\%), TIA (6.7\%), subarachnoid hemorrhage $(2.8 \%)$, and cerebral venous thrombosis $(0.2 \%) .^{2}$ Stroke is also the most common cause of complex disability and an independent risk for dementia. ${ }^{3}$ The direct and indirect cost of stroke care is a considerable burden to both the society and patients' families.

Although some risk factors of stroke are nonmodifiable, such as older age and male sex, current known modifiable risk factors-hypertension, current smoking, high waist-tohip ratio, diet, regular physical activity, diabetes mellitus, alcohol consumption, psychological factors, cardiac causes, ApoB/Apo1 ratio-together account for more than $90 \%$ of population attributable risk for stroke. ${ }^{2,4}$ In addition to these risk factors, metabolic syndrome, dyslipidemia, and high low-density lipoprotein cholesterol have been identified as risk factors for intracranial atherosclerosis, an important mechanistic step in the pathogenesis of ischemic stroke. $^{5}$

Vegetarian diets have been documented to lower blood pressure (BP), glucose, and cholesterol, ${ }^{6-8}$ and reduce cardiovascular disease incidence and mortality. ${ }^{9,10}$ Other dietary patterns that emphasized plant foods have been shown to be protectively associated with stroke incidence. ${ }^{11-14}$ However, vegetarians tend to have low intake of vitamin $B_{12}$, and this could raise homocysteine, leading to an increase in risk of stroke. ${ }^{15}$ In addition, previous studies suggested that some animal protein may be beneficial for the prevention of hemorrhagic stroke ${ }^{16}$ or improved cerebral infarction mortality. ${ }^{17}$ The combined effect of the supposed harm of high homocysteine and the supposed benefits of low BP, glucose, and cholesterol (unique aspect of vegetarian diets) on stroke disease incidence is unclear. A previous pool analysis showed that the association between vegetarian diet and cerebrovascular mortality trends toward protection in men (hazard ratio [HR], 0.77; 95\% confidence interval [CI], 0.57, 1.02) but not in women ( $\mathrm{HR}, 0.98 ; 95 \% \mathrm{CI}, 0.80,1.20)$, though overall association is heterogeneous in different studies. ${ }^{18}$ Moreover, many studies looking into diet and stroke incidence tend to lack information on BP, glucose, and cholesterol, making it difficult to examine whether the effect of diet is mediated through these relevant risk factors.
We investigated the incidence of overall, ischemic, and hemorrhagic stroke in vegetarians and nonvegetarians in 2 prospective cohorts in Taiwan. In addition, we explored whether the association between vegetarian diet and stroke risk is modified by vitamin $\mathrm{B}_{12}$ intake. We hypothesized that a vegetarian diet protects against stroke through reduction of metabolic risk factors and that this effect would be particularly strong among vegetarians with adequate intake of vitamin $B_{12}$.

\section{Methods}

\section{Standard protocol approvals, registrations, and patient consents}

The 2 cohorts were recruited in different periods and both were approved by the institutional review board at the Buddhist Dalin Tzu Chi General Hospital. All participants gave written informed consent.

The study was retrospectively registered on March 20, 2018. The protocol is available at clinicaltrials.gov (unique identifier: NCT03470584).

\section{Study design and population}

The 2 cohort studies, Tzu Chi Health Study (cohort 1) and Tzu Chi Vegetarian Study (cohort 2), were prospectively followed up to identify stroke events through linkage to the National Health Insurance Research Database (NHIRD).

Most of the participants were Tzu Chi volunteers, Buddhists who commit themselves to a variety of community services, ranging from charity to environmental protection. All volunteers went through at least 2 years of training to assure they know the core value of Tzu Chi and quit smoking and drinking alcohol before becoming certified as Tzu Chi volunteers. Vegetarianism is encouraged in this population as a means to promote planetary health and animal welfare. About $30 \%$ of the volunteers were full-time vegetarians in both cohorts.

\section{Tzu Chi Health Study (cohort 1)}

Cohort 1 recruited 6,002 participants (77\% certified Tzu Chi volunteers and $23 \%$ their families or individuals who came to the Buddhist Dalin Tzu Chi Hospital for routine health examination) from 2007 to 2009.

All participants received a comprehensive health examination at baseline and were interviewed on a questionnaire for demographics, medical history, lifestyle habits (smoking, alcohol, 
leisure time physical activities), and diet (a questionnaire on vegetarian dietary practice [type and duration] and a quantitative food frequency questionnaire [FFQ]). This FFQ (consisting of $64 \mathrm{food} /$ food groups with an additional section on use of supplements) had been validated among Tzu Chi volunteers and showed good reliability and validity; the correlation between FFQ-assessed vitamin $B_{12}$ and serum vitamin $B_{12}$ level is $0.41{ }^{19}$ Baseline fasting lipid profile, glucose, and creatinine were assessed through the INTEGRA 800 system (Roche Diagnostics, Indianapolis, IN; imprecision: $<2.5 \%$ coefficient of variation). Estimated glomerular filtration rate (eGFR) was calculated using the Chronic Kidney Disease Epidemiology Collaboration. BPs were measured using the VP 1000 System (Colin Co., Ltd., Komaki, Japan). A subgroup of 1,528 cohort members, selected at a ratio of 2:1 for nonvegetarians:vegetarians, contributed additional blood samples for analysis of homocysteine, vitamin $B_{12}$, and folate. Serum folate and vitamin $B_{12}$ were assessed by a commercial RIA kit (Siemens, Malvern, PA). Serum homocysteine was assessed using ADIVA Centuaur Immunoassay System (Siemens Healthcare Diagnostics Inc., Tarrytown, NY).

\section{Tzu Chi Vegetarian Study (cohort 2)}

Cohort 2 consists of 12,062 participants (average age 50 years) recruited from Tzu Chi Foundation branches throughout Taiwan in 2005. Community volunteer leaders in Tzu Chi sites throughout Taiwan assisted in distributing the questionnaire to volunteer members in their communities. The study questionnaire used in cohort 2 was similar to that of cohort 1 , except the FFQ had only 57 items, with frequency but no portion size. However, only cohort 1 had anthropometric and biochemical measurements. Cohort 2 lacked such data.

\section{Exposure assessment}

Vegetarian status was defined by avoidance of meat and fish in face to face confirmation by trained interviewers and in the FFQ in cohort 1 . As for cohort 2, the diet section includes a question inquiring whether the participant was a vegetarian or a nonvegetarian. Participants who identified themselves as vegetarians in this question and reported consuming no meat and fish in the FFQ were classified as vegetarians; those who self-identified as nonvegetarians or reported any consumption of meat or fish within the previous year (in the FFQ) were classified as nonvegetarians.

\section{Disease ascertainment}

The cohort data (including demographics and baseline diet and lifestyle) were linked to the NHIRD by a unique identification number under official legal permission. Incident stroke events and other baseline comorbidities were ascertained through NHIRD by the ICD-9-CM.

The National Health Insurance Program (NHIP) is a universal health care program implemented on March 1, 1995, in Taiwan and covers nearly $100 \%$ of Taiwan's population (nhird.nhri.org. tw/en/index.html). The NHIRD contains all medical claim records reimbursed by the NHIP and demographic information, such as dates of birth, sex, and clinical diagnoses, which are based on ambulatory clinical visit and inpatient care claims. The data are located in the Health and Welfare Data Science Center (HWDC) and belong to the Ministry of Health in Taiwan.

Incident stroke cases and stroke subtypes were prospectively identified during the 10-year follow-up (up to end of 2014) by the primary diagnosis in the hospitalization records. ${ }^{20}$ ICD-9$\mathrm{CM}$ codes were used to identify disease conditions: total stokes (430-438), hemorrhagic stroke (430-432), and ischemic stroke (433-434).

Other underlying comorbidities selected based on prior studies and suspected relationships were also considered: diabetes (250), hypertension (401-405), ischemic heart disease (410-414), and dyslipidemia (272). As the first diagnosis code may be given for further advanced examinations for ruling out purposes, presence of diseases was ascertained by having at least 2 diagnosis records of certain disease in order to prevent false-positives.

\section{Exclusion criteria}

We excluded participants who had no NHIRD data, age $<20$ years, those with missing values for covariates, and those with any stroke records in inpatient or outpatient database at baseline (the inclusion and exclusion scheme of the 2 cohorts is detailed in the figure).

\section{Statistical analysis}

Baseline descriptive statistics were compared between vegetarian and nonvegetarian groups using independent $t$ test and $\chi^{2}$ test. Intakes of foods and nutrients in vegetarians and nonvegetarians were compared using Wilcoxon rank-sum test (nonnormal distribution) and $\chi^{2}$ test. Wilcoxon rank-sum test and $\chi^{2}$ tests were used to compare serum homocysteine, vitamin $\mathrm{B}_{12}$, and folate between 406 vegetarians and 877 nonvegetarians.

Cox proportional hazards regression was used to examine the association between diet and stroke risk. Age rather than time on study was used as the time scale, as this is recommended by several statistical methodology articles as more appropriate in dealing with aging-related outcomes. ${ }^{21,22}$ For both cohorts, our analyses include crude model, sex-adjusted model, main model (adjusted for sex, education, body mass index [BMI] [only cohort 1], and lifestyle variables [smoking, alcohol drinking habits, betel nut use, and leisure time physical activities]), and final model (adjusted for factors in main model and relevant comorbidities, including hypertension, dyslipidemia, diabetes mellitus, and ischemic heart disease). We included a sensitivity analysis for cohort 1 , in which baseline measurements of systolic BP (SBP), fasting glucose, total cholesterol, and triglycerides were included instead of hypertension, diabetes, and dyslipidemia.

We further examined the relationship between diet and subtypes of stroke-ischemic and hemorrhagic - by competing risk analysis with cause-specific hazard function. ${ }^{23}$ 


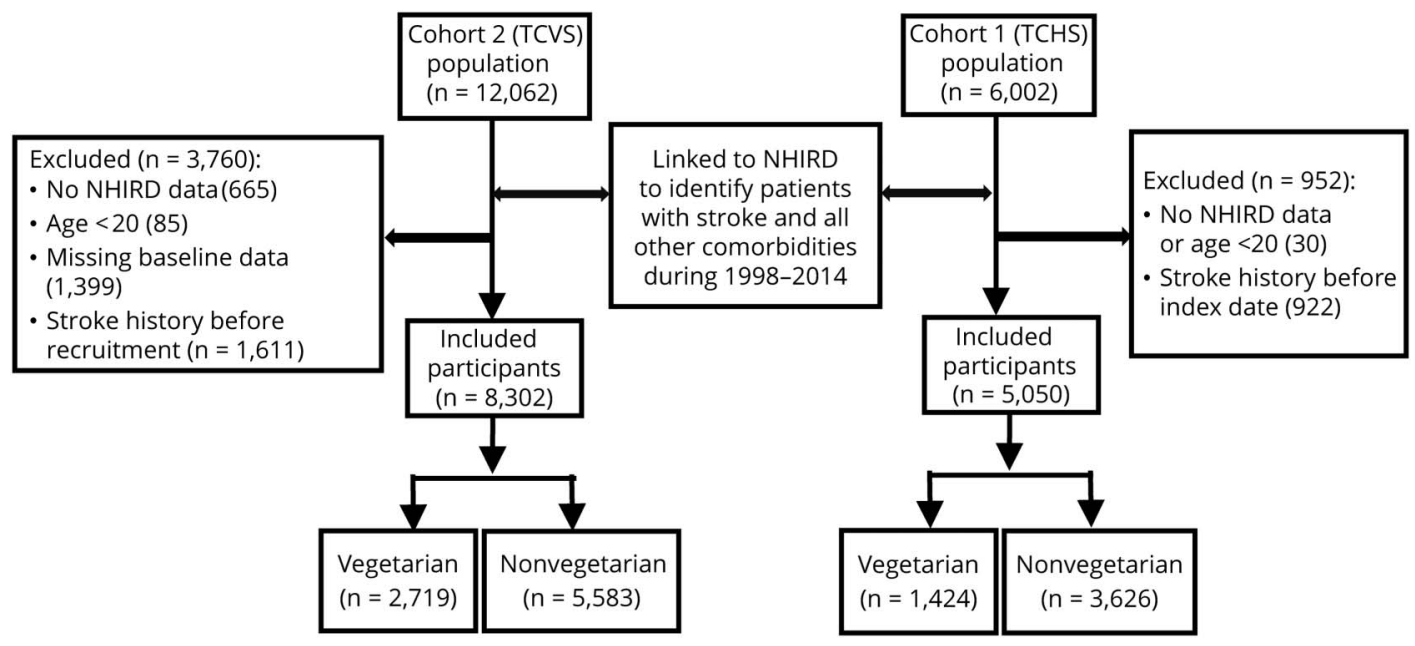

NHIRD = National Health Insurance Research Database; TCHS = Tzu Chi Health Study; TCVS = Tzu Chi Vegetarian Study.

Finally, we conducted a subgroup analysis by vitamin $B_{12}$ intake using the cut point of $2.4 \mu \mathrm{g}$, the recommended dietary allowance (RDA) for vitamin $B_{12}$ in Taiwan. Whether the effect of vegetarian diet on stroke risk is modified by vitamin $B_{12}$ was assessed by $p$ value of product between vegetarian diet and vitamin $\mathrm{B}_{12}$ in the Cox regression model.

All analyses were performed using SAS, version 9.4 (SAS Institute Inc., Cary, NC) within HWDC.

\section{Data availability}

All the dataset is located in the HWDC. As per local law and regulation, NHIRD data can only be accessed and analyzed within the facility of the HWDC. To access these data, one needs to submit an application to the HWDC of the Ministry of Health in Taiwan. All supplementary data are available at doi.org/10.5061/dryad.x95x69pcz.

\section{Results}

\section{Baseline characteristics of the 2 cohorts}

The baseline characteristics for the 2 cohorts are presented in table 1. Vegetarians had a higher proportion of women, neversmokers, and never-alcohol drinkers, and a lower proportion with elevated SBP (cohort 1) or hypertension (cohort 2), dyslipidemia, regular leisure time physical activities, and low education levels. The mean age was slightly higher in vegetarians, while BMI, cholesterol, and glucose were lower in vegetarians. Only $1.1 \%$ of vegetarians and $1.5 \%$ of nonvegetarians had abnormal renal function (eGFR $<60 \mathrm{~mL} / \mathrm{min} / 1.73 \mathrm{~m}^{2}$ ).

\section{Food and nutrient intakes estimated by FFQ}

Table 2 shows the intake of foods and nutrients (median and interquartile range) in nonvegetarians and vegetarians in cohort 1 . Vegetarians consumed more soy, vegetables, and nuts, and less dairy compared with nonvegetarians, while egg consumption was similar in both groups. For nutrients, vegetarians consumed more dietary fibers and plant protein, but less animal protein, fat, and vitamin $B_{12}$. The proportion with inadequate intakes of vitamin $\mathrm{B}_{12}$ was higher in vegetarians (64\%) than in nonvegetarians (33\%). The findings were similar when foods and nutrients were standardized to $2,000 \mathrm{kcal}$, as shown in table e-1 (doi.org/10.5061/dryad.x95x69pcz).

\section{Serum homocysteine, vitamin $B_{12}$, and folate concentration}

In selected participants with serum homocysteine, vitamin $B_{12}$, and folate data available (406 vegetarians and 877 nonvegetarians) within cohort 1 , vegetarians had lower serum vitamin $B_{12}$ and higher serum folate and homocysteine, as indicated by median (table 3 ). Additional comparison of least square mean of these biomarkers (between vegetarians and nonvegetarians), with adjustment of sex, smoking, and alcohol drinking by general linear model, showed no substantial change (data not shown). Vegetarians were more likely to have elevated homocysteine ( $25 \%$ of vegetarians vs $8 \%$ of nonvegetarians had homocysteine $\geq 14 \mu \mathrm{mol} / \mathrm{L})$ and vitamin $B_{12}$ deficiency $(26 \%$ of vegetarians vs $1 \%$ of nonvegetarians had serum vitamin $B_{12}<200 \mathrm{pg} / \mathrm{mL}$ ), as shown in table 3. Folate deficiency was rare, and the numbers were too small to be released as per regulation of the HWDC.

\section{Association between vegetarian diet and stroke incidence in cohort 1}

There were 54 incident stroke events in the 30,797 personyears of follow-up. Overall stroke incidence in vegetarians was lower than in nonvegetarians (1.16 [0.62-2.15] vs 1.99 [1.48-2.67] per 1,000 person-years). As shown in table 4, vegetarians (vs nonvegetarians) had only about half of the risk of overall stroke throughout various models. SBP and fasting glucose were associated with risk (in sensitivity analysis; table e-2, doi.org/10.5061/dryad.x95x69pcz). 
Table 1 Baseline characteristics of vegetarians and nonvegetarians in cohort 1 (Tzu Chi Health Study [TCHS]) and cohort 2 (Tzu Chi Vegetarian Study [TCVS])

\begin{tabular}{|c|c|c|c|c|c|c|}
\hline \multirow[b]{2}{*}{ Characteristic } & \multicolumn{3}{|l|}{ Cohort 1 (TCHS) } & \multicolumn{3}{|l|}{ Cohort 2 (TCVS) } \\
\hline & $\begin{array}{l}\text { Nonvegetarians } \\
(n=3,626)\end{array}$ & $\begin{array}{l}\text { Vegetarians } \\
(n=1,424)\end{array}$ & $p$ Value & $\begin{array}{l}\text { Nonvegetarians } \\
(n=5,583)\end{array}$ & $\begin{array}{l}\text { Vegetarians } \\
(n=2,719)\end{array}$ & $p$ Value \\
\hline Age, y, mean (SD) & $51.7(10.5)$ & $53.8(9.03)$ & $<0.01$ & $49.2(9.6)$ & $50.1(9.3)$ & $<0.01$ \\
\hline Male, \% & 47.2 & 25.1 & 0.05 & 40.5 & 27.9 & $<0.01$ \\
\hline Education level, $\%$ & & & $<0.01$ & & & $<0.01$ \\
\hline Elementary school or less & 20.5 & 28.4 & & 18.5 & 22.3 & \\
\hline Secondary school & 48.6 & 49.9 & & 54.0 & 51.2 & \\
\hline College or higher & 30.9 & 21.7 & & 27.6 & 26.5 & \\
\hline Ever smoking, \% & 21.2 & 8.5 & $<0.01$ & 16.7 & 10.2 & $<0.01$ \\
\hline Ever alcohol drinking, \% & 18.0 & 8.1 & $<0.01$ & 15.2 & 11.0 & $<0.01$ \\
\hline Ever betel nut, \% & 7.7 & 3.0 & $<0.01$ & 6.3 & 4.8 & $<0.01$ \\
\hline $\begin{array}{l}\text { Leisure time physical } \\
\text { activities, \% }\end{array}$ & 68.7 & 64.7 & $<0.01$ & 84.7 & 80.8 & $<0.01$ \\
\hline Hypertension, \% & 14.5 & 14.2 & 0.79 & 11.5 & 9.4 & $<0.01$ \\
\hline Diabetes mellitus, \% & 5.8 & 4.6 & 0.09 & 13.6 & 11.8 & 0.05 \\
\hline Dyslipidemia, \% & 8.5 & 5.7 & $<0.01$ & 6.4 & 3.8 & $<0.01$ \\
\hline Ischemic heart disease, \% & 3.9 & 3.7 & 0.81 & 2.9 & 2.5 & 0.29 \\
\hline SBP, mean (SD) & $126(16.4)$ & $125(17.0)$ & 0.02 & & & \\
\hline Elevated blood pressure, \% & 29.8 & 23.4 & $<0.01$ & & & \\
\hline Fasting glucose, mean (SD) & $94.3(20.0)$ & $92.3(15.9)$ & $<0.01$ & & & \\
\hline Impaired fasting glucose, $\%$ & 19.3 & 14.0 & $<0.01$ & & & \\
\hline Total cholesterol, mean (SD) & $196(36.4)$ & $180(33.9)$ & $<0.01$ & & & \\
\hline HDL, mean (SD) & $54.4(14.8)$ & $52.4(13.7)$ & $<0.01$ & & & \\
\hline LDL, mean (SD) & $129(33.3)$ & $115(29.4)$ & $<0.01$ & & & \\
\hline Elevated total cholesterol, \% & 43.5 & 24.9 & $<0.01$ & & & \\
\hline Triglycerides, mean (SD) & $115(83.4)$ & $113(77.2)$ & 0.48 & & & \\
\hline Elevated triglycerides, \% & 19.8 & 19.7 & 0.91 & & & \\
\hline BMI, mean (SD) & $23.9(3.3)$ & $23.0(3.1)$ & $<0.01$ & & & \\
\hline $\mathrm{BMI} \geq 24, \%$ & 45.7 & 33.5 & $<0.01$ & & & \\
\hline Serum creatinine, mean (SD) & $0.71(0.22)$ & $0.63(0.23)$ & $<0.01$ & & & \\
\hline eGFR, mean (SD) & $103(15.1)$ & $104.7(13.8)$ & $<0.01$ & & & \\
\hline eGFR $<60, \%$ & 1.5 & 1.1 & 0.26 & & & \\
\hline
\end{tabular}

Abbreviations: $\mathrm{BMI}=$ body mass index; DBP = diastolic blood pressure; eGFR = estimated glomerular filtration rate; $\mathrm{HDL}=$ high-density lipoprotein cholesterol; $\mathrm{LDL}=$ low-density lipoprotein cholesterol; SBP = systolic blood pressure.

Elevated blood pressure is defined as SBP $\geq 120$ or DBP $\geq 80 \mathrm{~mm} \mathrm{Hg}$, or both. Impaired fasting glucose is defined as $\geq 100 \mathrm{mg} / \mathrm{dL}$. Elevated total cholesterol is defined as $\geq 200 \mathrm{mg} / \mathrm{dL}$. Elevated triglycerides is defined as $\geq 150 \mathrm{mg} / \mathrm{dL}$.

When examining specific stroke subtypes, vegetarians had $74 \%$ lower ischemic stroke risk throughout different models. Individuals with diabetes had about 3 -fold risk of ischemic stroke when compared with nondiabetic patients (table e-2, doi.org/ $10.5061 /$ dryad.x95x69pcz). In the sensitivity analysis, the protective association between vegetarian diet and ischemic stroke slightly attenuated after adjusting for fasting glucose, SBP, triglycerides, and total cholesterol (HR, 0.28; 0.08-0.96). Analysis of hemorrhagic stroke was not performed due to a small number of events $(n=8)$ in this cohort. 
Table 2 Dietary intakes of vegetarians and nonvegetarians based on food frequency questionnaires in cohort 1 (Tzu Chi Health Study)

\begin{tabular}{|c|c|c|c|}
\hline Foods and nutrients & $\begin{array}{l}\text { Nonvegetarians } \\
(n=3,626), \\
\text { median } \\
\text { (IQR) }\end{array}$ & $\begin{array}{l}\text { Vegetarians } \\
(n=1,424) \text {, } \\
\text { median } \\
(\text { IQR) }\end{array}$ & $p$ Value \\
\hline Meat, servings & $0.6(0.2,1.6)$ & - & - \\
\hline Fish, servings & $0.5(0.1,1.1)$ & - & - \\
\hline Eggs, servings & $0.3(0.1,0.6)$ & $0.3(0.1,0.5)$ & $<0.01$ \\
\hline Dairy, servings & $0.3(0,0.9)$ & $0.2(0,0.7)$ & $<0.01$ \\
\hline Soy, servings & $1.0(0.5,1.7)$ & $1.5(0.8,2.5)$ & $<0.01$ \\
\hline Vegetables, servings & $3.8(2.3,5.7)$ & $4.5(2.9,6.8)$ & $<0.01$ \\
\hline Fruits, servings & $1.0(0.5,2.0)$ & $1.0(0.6,2.0)$ & 0.07 \\
\hline Nuts, servings & $0.2(0.0,0.5)$ & $0.3(0.1,1.0)$ & $<0.01$ \\
\hline Energy, kcal & $\begin{array}{l}1740 \\
(1,306,2,297)\end{array}$ & $\begin{array}{l}1705 \\
(1,288,2,247)\end{array}$ & 0.11 \\
\hline Protein (\% energy) & $13(12,15)$ & $12(11,13)$ & $<0.01$ \\
\hline Animal protein, $\mathrm{g}$ & $19(11,31)$ & $4(2,7)$ & $<0.01$ \\
\hline Plant protein, $\mathrm{g}$ & $38(28,51)$ & $46(34,61)$ & $<0.01$ \\
\hline Fat (\% energy) & $27(22,33)$ & $25(19,30)$ & $<0.01$ \\
\hline Saturate fat, $\mathrm{g}$ & $11(7,17)$ & $9(6,13)$ & $<0.01$ \\
\hline $\begin{array}{l}\text { Monounsaturated } \\
\text { fat, } g\end{array}$ & $16(10,25)$ & $12(8,19)$ & $<0.01$ \\
\hline $\begin{array}{l}\text { Polyunsaturated } \\
\text { fat, } g\end{array}$ & $12(7,20)$ & $11(6,18)$ & $<0.01$ \\
\hline $\begin{array}{l}\text { Carbohydrate } \\
\text { (\% energy) }\end{array}$ & $60(53,67)$ & $64(58,70)$ & $<0.01$ \\
\hline Dietary fiber, $\mathrm{g}$ & $19(14,26)$ & $23(16,30)$ & $<0.01$ \\
\hline Vitamin $B_{12}, \mu g$ & $4.0(1.9,9.5)$ & $1.4(0.6,5.2)$ & $<0.01$ \\
\hline $\begin{array}{l}\text { Vitamin } B_{12} \text { intake } \\
<2.4 \mu \mathrm{g}, \%\end{array}$ & 33.3 & 64.0 & $<0.01$ \\
\hline $\begin{array}{l}\text { Use of vitamin } B_{12} \\
\text { supplement, } \%\end{array}$ & 36.8 & 40.0 & 0.04 \\
\hline
\end{tabular}

Abbreviation: IQR = interquartile range.

A serving of meat, fish, eggs, or soy is defined as $7 \mathrm{~g}$ protein; a serving of dairy is defined as $8 \mathrm{~g}$ protein; a serving of vegetable is defined as $100 \mathrm{~g}$; a serving of fruit is defined as $15 \mathrm{~g}$ carbohydrates; a serving of nuts is defined as $45 \mathrm{kcal}$.

\section{Association between vegetarian diet and stroke incidence in cohort 2}

There were 121 incident stroke events in the 76,797 personyears of follow-up. Stroke incidence in vegetarians was lower than in nonvegetarians $(0.95$ [95\% CI, 0.64-1.42] vs 1.88 [95\% CI, 1.54-2.29] per 1,000 person-years). Compared with nonvegetarians, vegetarians experienced only about half of the risk of overall stroke through all the models considered, as shown in table 4. Hypertension is an independent risk factor (HR, 1.82; 95\% CI, 1.17-2.85), as shown in table e-3 (doi.org/10.5061/dryad.x95x69pcz).
Table 3 Serum homocysteine, vitamin $\mathrm{B}_{12}$, and folate concentration in 877 nonvegetarians and 406 vegetarians in cohort 1 (Tzu Chi Health Study)

\begin{tabular}{|c|c|c|c|}
\hline & Nonvegetarians & Vegetarians & $p$ Value \\
\hline \multicolumn{4}{|l|}{$\begin{array}{l}\text { Homocysteine, } \\
\mu \mathrm{mol} / \mathrm{L}\end{array}$} \\
\hline Median (IQR) & $9.3(7.9,11.3)$ & $10.6(8.7,14.0)$ & $<0.01^{a}$ \\
\hline$<12, \%$ & 82 & 63 & $<0.01^{\mathrm{b}}$ \\
\hline$\geq 12,<14, \%$ & 10 & 12 & \\
\hline$\geq 14, \leq 20, \%$ & 6 & 20 & \\
\hline$>20, \%$ & 2 & 5 & \\
\hline
\end{tabular}

Vitamin $\mathbf{B}_{12}$,

$\mathrm{pg} / \mathrm{mL}$

\begin{tabular}{llll}
\hline Median (IQR) & $487(374,653)$ & $280(196,407)$ & $<0.01^{\mathrm{a}}$ \\
\hline$<\mathbf{2 0 0} \%$ & 1 & 26 & $<0.01^{\mathrm{b}}$ \\
\hline$\geq \mathbf{2 0 0},<\mathbf{3 0 0} \%$ & 10 & 28 & \\
\hline$\geq \mathbf{3 0 0}, \%$ & 89 & 46 &
\end{tabular}

\section{Folate,}

$\mathrm{ng} / \mathrm{mL}$

\begin{tabular}{llll} 
Median (IQR) $\quad 10.6(7.9,14.0)$ & $13.4(10.1,17.4) \quad<0.01^{\mathrm{a}}$ \\
\hline
\end{tabular}

Abbreviation: IQR = interquartile range.

a $p$ Value calculated using Wilcoxon rank-sum test.

${ }^{b} p$ Value calculated using $\chi^{2}$ test.

For ischemic stroke, vegetarians consistently had about $60 \%$ lower risk than nonvegetarians throughout all models. Ever smoking (vs never) appeared to be independently associated with a nearly 2.5 times risk (table e-3, doi.org/10.5061/dryad. $\mathrm{x} 95 \mathrm{x} 69 \mathrm{pcz}$ ). Having hypertension (vs none) was also associated with greater than 2-fold risk of ischemic stroke (table e-3, doi.org/10.5061/dryad.x95x69pcz).

For hemorrhagic stroke, vegetarians also experienced about $65 \%$ lower risk than nonvegetarians, and this finding appeared consistent throughout all models (table 4).

\section{Subgroup analysis by vitamin $B_{12}$ intakes}

Our subgroup analysis by vitamin $B_{12}$ intake is shown in table 5. Inverse association between vegetarian diet and lower overall stroke was found in the subgroup with inadequate vitamin $B_{12}$ intake $(<2.4 \mu \mathrm{g})$ but not in those with adequate vitamin $B_{12}$ intake $(\geq 2.4 \mu \mathrm{g}), p$ interaction $=0.046$. Baseline characteristic and dietary intakes of the subgroups as assessed by FFQ are presented in tables e- 4 and e- 5 (doi. org/10.5061/dryad.x95x69pcz), respectively. Vegetarians with adequate vitamin $B_{12}$ intake consumed more animal protein (median 5 vs $3 \mathrm{~g}$ ) and were more likely to take vitamin $B_{12}$ supplements ( $96 \%$ vs $8.5 \%$ ) than those with inadequate vitamin $B_{12}$ intake. 
Table 4 Association between vegetarian diet (vs nonvegetarian diet) and stroke incidence in cohort 1 (Tzu Chi Health Study) and cohort 2 (Tzu Chi Vegetarian Study)

\begin{tabular}{|c|c|c|c|c|c|c|c|}
\hline & $\begin{array}{l}\text { Nonvegetarian, } \\
\text { cases/n }\end{array}$ & $\begin{array}{l}\text { Vegetarian, } \\
\text { cases/n }\end{array}$ & $\begin{array}{l}\text { Crude } \\
\text { model }\end{array}$ & Sex-adjusted & $\begin{array}{l}\text { Main } \\
\text { model }\end{array}$ & $\begin{array}{l}\text { Final } \\
\text { model }\end{array}$ & $\begin{array}{l}\text { Sensitivity } \\
\text { analysis }\end{array}$ \\
\hline \multicolumn{8}{|l|}{ Cohort 1} \\
\hline Overall stroke & $44 / 3,626$ & $10 / 1,424$ & $\begin{array}{l}0.50 \\
(0.25,1.01)\end{array}$ & $\begin{array}{l}0.53 \\
(0.26,1.06)\end{array}$ & $\begin{array}{l}0.55 \\
(0.27,1.11)\end{array}$ & $\begin{array}{l}0.51 \\
(0.25,1.06)\end{array}$ & $\begin{array}{l}0.54 \\
(0.26,1.13)\end{array}$ \\
\hline Ischemic stroke & $28 / 3,626$ & $3 / 1,424$ & $\begin{array}{l}0.25 \\
(0.08,0.83)\end{array}$ & $\begin{array}{l}0.26 \\
(0.08,0.86)\end{array}$ & $\begin{array}{l}0.26 \\
(0.08,0.88)\end{array}$ & $\begin{array}{l}0.26 \\
(0.08,0.88)\end{array}$ & $\begin{array}{l}0.28 \\
(0.08,0.96)\end{array}$ \\
\hline \multicolumn{8}{|l|}{ Cohort 2} \\
\hline Overall stroke & $97 / 5,583$ & $24 / 2,719$ & $\begin{array}{l}0.47 \\
(0.30,0.74)\end{array}$ & $\begin{array}{l}0.50 \\
(0.32,0.78)\end{array}$ & $\begin{array}{l}0.51 \\
(0.33,0.80)\end{array}$ & $\begin{array}{l}0.52 \\
(0.33,0.82)\end{array}$ & \\
\hline Ischemic stroke & $46 / 5,583$ & $8 / 2,719$ & $\begin{array}{l}0.33 \\
(0.16,0.70)\end{array}$ & $\begin{array}{l}0.37 \\
(0.17,0.78)\end{array}$ & $\begin{array}{l}0.39 \\
(0.18,0.84)\end{array}$ & $\begin{array}{l}0.41 \\
(0.19,0.88)\end{array}$ & \\
\hline $\begin{array}{l}\text { Hemorrhagic } \\
\text { stroke }\end{array}$ & $24 / 5,583$ & $4 / 2,719$ & $\begin{array}{l}0.32 \\
(0.11,0.91)\end{array}$ & $\begin{array}{l}0.34 \\
(0.12,1.00)\end{array}$ & $\begin{array}{l}0.35 \\
(0.12,1.02)\end{array}$ & $\begin{array}{l}0.34 \\
(0.12,1.00)\end{array}$ & \\
\hline
\end{tabular}

Values are hazard ratios (95\% confidence intervals) estimated by Cox regression with age as time scale. Sex-adjusted model adjusted for sex only. Main model adjusted for sex, smoking (ever vs never), alcohol drinking (ever vs never), betel nut (ever vs never), leisure time physical activities (yes vs no), and education. Final model adjusted for all variables in main model, plus hypertension, diabetes mellitus, dyslipidemia, ischemic heart disease, and body mass index ( $\geq 24$ vs $<24$, for cohort 1 only). Sensitivity analysis adjusted for all variables in main model, plus body mass index ( $\geq 24$ vs $<24)$, systolic blood pressures, fasting glucose, total cholesterol, and triglycerides.

\section{Discussion}

Our prospective cohort studies showed that a vegetarian diet is associated with a lower risk of both ischemic stroke and hemorrhagic stroke. Potential effect modification by dietary vitamin $B_{12}$ intake was found for overall stroke.

The most important modifiable risk factor for stroke is hypertension, accounting for $47.9 \%, 45.7 \%$, and $56.4 \%$ of population attributable risk for overall, ischemic, and hemorrhagic strokes, respectively, in the INTERSTROKE study. ${ }^{4}$ In our cohort studies, vegetarians had lower baseline prevalence of elevated BPs (23 vs $30 \%$ in cohort 1 ) and hypertension ( 9.4 vs $11.5 \%$ in cohort 2 ) than nonvegetarians. The inverse association between vegetarian diet and BPs in our study is consistent with a previous systematic review and meta-analysis ${ }^{8}$ and a prospective study. ${ }^{24}$ The health benefits of a plant-based diet for BP control have been so well-documented that the
Dietary Approach to Stop Hypertension (DASH) - the gold standard diet for hypertension management-emphasizes increased consumption of healthy plant-based food and minimization of red and processed meat, and this diet has also been shown to lower the risk for overall stroke ${ }^{12}$ and ischemic stroke. ${ }^{13}$ The PREDIMED trial that randomized 7,447 highrisk participants into the Mediterranean diet (also a plantbased pattern) vs control diet found that the Mediterranean diet reduced the risk of overall stroke by $42 \%$ compared with the control diet, supporting the consistent beneficial effect of plant-based dietary patterns. ${ }^{11}$ Since the incidence of ischemic stroke is much higher than that of the hemorrhagic type globally and in Taiwan, the protective association between diet and overall stroke may be attributable to ischemic stroke.

In addition to hypertension, vegetarian diets have been shown to lower other important risk factors of ischemic stroke, including both low-density lipoprotein (LDL)-cholesterol and

Table 5 Subgroup analysis by vitamin $B_{12}$ intake on the association between vegetarian diet (vs nonvegetarian diet) and overall stroke incidence in cohort 1 (Tzu Chi Vegetarian Study)

\begin{tabular}{lllll}
\hline & $\begin{array}{l}\text { Nonvegetarians, } \\
\text { cases/n }\end{array}$ & $\begin{array}{l}\text { Vegetarians, } \\
\text { cases/n }\end{array}$ & HR (95\% Cl) & $\boldsymbol{p}$ interaction \\
\hline Vitamin $\mathbf{B}_{\mathbf{1 2}}$ intake $<\mathbf{2 . 4} \boldsymbol{\mu g} / \mathrm{d}$ & $20 / 1,208$ & $4 / 911$ & $0.27(0.09$, \\
& & $0.83)$ & 0.046 \\
\hline Vitamin $\mathbf{B}_{\mathbf{1 2}}$ intake $\mathbf{2} \mathbf{2 . 4} \boldsymbol{\mu g} / \mathrm{d}$ & $24 / 2,418$ & $6 / 513$ & $\begin{array}{l}0.99(0.38, \\
2.57)\end{array}$
\end{tabular}

Abbreviations: $\mathrm{Cl}=$ confidence interval; $\mathrm{HR}=$ hazard ratio

HR is estimated using Cox regression, with age as time scale, and adjustment for sex (men vs women), smoking (ever vs never), alcohol drinking (ever vs never), betel nut (ever vs never), leisure time physical activities (yes vs no), education (elementary or less vs secondary vs college or higher), hypertension (yes vs no), diabetes (yes vs no), dyslipidemia (yes vs no), ischemic heart diseases (yes vs no), body mass index ( $\geq 24$ vs $<24$ ), systolic blood pressures, fasting glucose, total cholesterol, and triglycerides. 
diabetes. ${ }^{6,25}$ The mean concentration of LDL-cholesterol in our vegetarians was $11 \%$ lower than in the nonvegetarians (115 vs $129 \mathrm{mg} / \mathrm{dL}$ ). This may contribute to significant protection, as a $10 \%$ reduction in LDL-cholesterol has been shown to reduce the relative risk of primary stroke by $13.5 \%$ in a meta-analysis of statin trials. ${ }^{26}$ Vegetarian dietary pattern in our population is characterized by higher intake of vegetables, soy, and nuts. These plant foods are major sources of antioxidants, $^{27}$ which could reduce oxidative stress known to impair endothelial function and induce atherosclerosis. ${ }^{28}$ Higher consumption of plant protein, dietary fiber, and antioxidants have all been shown to protect against stroke in prospective studies. ${ }^{29-31}$

In our study, the beneficial association between vegetarian diet and ischemic stroke was attenuated only slightly after controlling for known cardiometabolic mediators (BPs, glucose, and lipid) or comorbidities (hypertension, diabetes, dyslipidemia, and ischemic heart diseases). This suggests that additional protective mechanisms may exist. Carnitine from meat and choline from eggs not only provide substrates for but also shift gut microbial community to enhance the production of trimethylamine $\mathrm{N}$-oxide (TMAO), ${ }^{32}$ an atherosclerotic factor that has been shown to induce platelet hyperreactivity and thrombosis $^{33}$ and increase stroke incidence. ${ }^{34}$ Vegetarians' chance of being a high TMAO producer is only one-tenth that of nonvegetarians, ${ }^{35}$ and this may exert additional protection on top of the traditional risk factors for stroke.

The strong inverse association between vegetarian diet and hemorrhagic stroke in our study contrasts the results of the recently published EPIC-Oxford study. ${ }^{36}$ Buddhist vegetarians in our population and the EPIC-Oxford vegetarians differ in alcohol consumption and use of allium vegetables. Nearly $80 \%$ of vegetarians in the EPIC-Oxford study drink varying degrees of alcohol but most participants in our studies avoid alcohol (about $6 \%$ of nonvegetarians and fewer than $1 \%$ of vegetarians were current drinkers at baseline). Alcohol consumption (as measured by $\gamma$-glutamyl transferase) has been suggested in a previous cohort study to modify the effect of low serum cholesterol (typical of vegetarians) on hemorrhagic stroke risk. ${ }^{37}$ The EPICCVD case cohort study also found a clear trend between increased alcohol consumption and stroke risk. ${ }^{38}$ While Western vegetarians use ample amounts of garlic and other allium vegetables, Asian Buddhist vegetarians avoid these vegetables. Allium vegetables are major sources of alliin/allicin, known to inhibit platelet function and induce bleeding. ${ }^{39}$ Whether these differences drive the discrepant findings warrants further investigation.

Hypertension is the most important risk factor for hemorrhagic stroke. ${ }^{4}$ Vegetarian diet and other plant-based diets such as DASH and Mediterranean dietary patterns effectively reduce BPs, as evidenced by systematic review and meta-analysis of randomized controlled trials. ${ }^{8,40}$ Previous cohort studies found that the highest vs lowest adherence to DASH and Mediterranean diet were associated with a $10 \%-20 \%$ reduction in hemorrhagic stroke. ${ }^{12,14,41}$ In addition to the BP-lowering effect common to most plant-based diets, a vegetarian diet may have the additional advantage of reduced TMAO, ${ }^{35}$ and TMAO has been positively associated with hemorrhagic stroke risk among hypertensive individuals in a recent nested casecontrol study. ${ }^{34}$

Many studies reporting inverse associations between intake of animal products and hemorrhagic stroke focus on a single food or nutrient. ${ }^{16,29,42}$ The effect of animal products or nutrients would be difficult to single out if they correlate highly with other protective plant foods. This is not uncommon in Asian cultures: in the Japanese Public Health Center-based prospective study, fish appears to be a marker for a healthy dietary pattern that simultaneously contains higher vegetables, fruits, soy, seaweeds, potato, and mushroom, derived from principal component analysis. ${ }^{43}$ The dietary cluster with the lowest fish and meat intake also contained the lowest vegetables and fruits in the Shanghai Women's Health Study. ${ }^{44}$

Despite higher homocysteine due to lower vitamin $\mathrm{B}_{12}$ intake, vegetarians did not have higher risk of overall, ischemic, or hemorrhagic strokes. A recent Mendelian randomization analysis and the Vitamins to Prevent Stroke (VITATOPS) trial both found that lowering of homocysteine is associated with only the small vessel subtype of ischemic stroke-which accounts for $37.7 \%$ of ischemic and $27.9 \%$ of overall stroke in Taiwan ${ }^{2}$-but not other subtypes (large artery or cardioembolic). ${ }^{41,45}$ The ICD-9-CM code could not distinguish to such detail and our studies would not have enough power to examine these detailed subtypes of ischemic stroke. In our study, the percentage of study participants with hyperhomocysteinemia (defined as $>20 \mu \mathrm{mol} / \mathrm{L}$ ) is low $(2 \%$ in nonvegetarians vs $5 \%$ in vegetarians), likely due to healthy overall lifestyle (nonsmoking, no alcohol drinking, adequate folate intake through high vegetable consumption), thus stroke risk attributable to hyperhomocysteinemia is probably low.

Interestingly, our study found that the inverse association between vegetarian diet and overall stroke risk is more pronounced in the subgroup consuming less than the RDA of vitamin $\mathrm{B}_{12}$, but not the subgroup with adequate intakes ( $p$ interaction $=0.046)$. Supplementation with high-dose cyanocobalamin (a form of vitamin $\mathrm{B}_{12}$ ) increases the risk of stroke among individuals with reduced renal function. ${ }^{46}$ Although we have no data on the type of vitamin $\mathrm{B}_{12}$ supplements (cyanocobalamin, methylcobalamin, or hydroxocobalamin) to clarify whether the attenuation of protection is related to cyanocobalamin, it is unlikely to be the major reason as the percent of vegetarians with abnormal renal function is small $(1.8 \%$ in vegetarians meeting the $R D A$ for vitamin $\left.B_{12}\right)$. Vitamin $B_{12}$ may be a surrogate marker for animal product consumption and this may account for some attenuation. Due to small sample size, this finding needs to be interpreted with caution and confirmed in other studies.

Our study has several strengths. First, the high proportion of vegetarians $(\sim 30 \%)$ enables us to examine the association 
between vegetarian diet and stroke risk with adequate power. Second, the high follow-up rate due to nearly complete coverage in the NHIRD prevents loss to follow-up and minimizes selection bias. Third, data on stroke-relevant metabolic risk factors, such as fasting blood glucose, total cholesterol, BPs, and BMI, were available. Fourth, the consistent results in both cohorts strengthen confidence in the finding. Finally, vegetarians and nonvegetarians were from the same religious group and abstain from alcohol and tobacco; this would minimize confounding associated with religion and related lifestyle, and strengthen internal validity, while providing a unique opportunity to examine the effect of plant-based diet among people who do not drink alcohol, which is rare in most Western studies.

Several limitations should be considered when interpreting these findings. First, diet was assessed only at baseline and change over time is possible, though this should attenuate the protective effect of the vegetarian diet-bias toward the null. Second, the definition of stroke is based on ICD-9-CM, not MRI findings, and there are no data on detailed subtypes within ischemic stroke or hemorrhagic stroke. However, the stroke ICD-9-CM codes in NHIRD have been validated and demonstrated a $97 \%$ positive predictive value with specified coding. ${ }^{20}$ Third, the generalizability of study findings may be limited to populations that abstain from cigarettes and alcohol. The overall finding contrasts with that of the EPICOxford study but is consistent with previous studies on plant-based diets and stroke. ${ }^{1-14}$ Fourth, the small sample size made it difficult to evaluate the association between the vegetarian diet and stroke subtypes, or make conclusion on subgroup analysis and interaction. Finally, residual confounding may be present, despite extensive adjustments for potential confounding factors, due to the observational study design.

\section{Author contributions}

T.H.T.C., H.-R.C., and L.-Y.W. wrote the paper. L.-Y.W. and C.-C.C. conducted the statistical analysis. M.-N.L. and C.-L.L. designed the study and collected the baseline data.

\section{Study funding}

The establishment of the cohorts was supported by a grant from the Department of Health in Taiwan (DOH94-TD-F-113-044) and a grant from Buddhist Dalin Tzu Chi General Hospital (TCRD: 19605-02). The follow-up was supported by grants from the Buddhist Tzu Chi Medical Foundation (TCMMPSP104-08-02, TCMMP105-13-05, and TCMMP10604-01) and a grant from the Ministry of Science and Technology in Taiwan (108-2320-B-030-007). The funders played no role in study design, data collection, data analysis, data interpretation, or writing of the report.

\section{Disclosure}

The authors report no disclosures relevant to the manuscript. Go to Neurology.org/N for full disclosures.

\section{Publication history}

Received by Neurology March 5, 2019. Accepted in final form October 10, 2019.

\section{References}

1. Benjamin EJ, Virani SS, Callaway CW, et al. Heart disease and stroke statistics: 2018 update: a report from the American Heart Association. Circulation 2018;137: e67-e492.

2. Hsieh FI, Chiou HY. Stroke: morbidity, risk factors, and care in Taiwan. J Stroke 2014;16:59-64.

3. Pohjasvaara T, Erkinjuntti T, Ylikoski R, Hietanen M, Vataja R, Kaste M. Clinical determinants of poststroke dementia. Stroke 1998;29:75-81.

4. O'Donnell MJ, Chin SL, Rangarajan S, et al. Global and regional effects of potentially modifiable risk factors associated with acute stroke in 32 countries (INTERSTROKE): a case-control study. Lancet 2016;388:761-775.

5. Ma YH, Leng XY, Dong Y, et al. Risk factors for intracranial atherosclerosis: a systematic review and meta-analysis. Atherosclerosis 2019;281:71-77.

6. Wang F, Zheng J, Yang B, Jiang J, Fu Y, Li D. Effects of vegetarian diets on blood lipids: a systematic review and meta-analysis of randomized controlled trials. J Am Heart Assoc 2015;4:e002408.

7. Yokoyama Y, Barnard ND, Levin SM, Watanabe M. Vegetarian diets and glycemic control in diabetes: a systematic review and meta-analysis. Cardiovasc Diagn Ther 2014;4:373-382

8. Yokoyama Y, Nishimura K, Barnard ND, et al. Vegetarian diets and blood pressure: a meta-analysis. JAMA Intern Med 2014;174:577-587.

9. Crowe FL, Appleby PN, Travis RC, Key TJ. Risk of hospitalization or death from ischemic heart disease among British vegetarians and nonvegetarians: results from the EPIC-Oxford cohort study. Am J Clin Nutr 2013;97:597-603.

10. Orlich MJ, Singh PN, Sabate J, et al. Vegetarian dietary patterns and mortality in Adventist Health Study 2. JAMA Intern Med 2013;173:1230-1238.

11. Estruch R, Ros E, Salas-Salvado J, et al. Primary prevention of cardiovascular disease with a Mediterranean diet supplemented with extra-virgin olive oil or nuts. $\mathrm{N}$ Engl J Med 2018;378:e34.

12. Fung TT, Chiuve SE, McCullough ML, Rexrode KM, Logroscino G, Hu FB. Adherence to a DASH-style diet and risk of coronary heart disease and stroke in women. Arch Intern Med 2008;168:713-720.

13. Larsson SC, Wallin A, Wolk A. Dietary approaches to stop hypertension diet and incidence of stroke: results from 2 prospective cohorts. Stroke 2016;47: 986-990

14. Tektonidis TG, Akesson A, Gigante B, Wolk A, Larsson SC. A Mediterranean diet and risk of myocardial infarction, heart failure and stroke: a population-based cohort study. Atherosclerosis 2015;243:93-98.

15. Hankey GJ. B vitamins for stroke prevention. Stroke Vasc Neurol 2018;3:51-58

16. Sauvaget C, Nagano J, Allen N, Grant EJ, Beral V. Intake of animal products and stroke mortality in the Hiroshima/Nagasaki Life Span Study. Int J Epidemiol 2003;32: 536-543.

17. Sauvaget C, Nagano J, Hayashi M, Yamada M. Animal protein, animal fat, and cholesterol intakes and risk of cerebral infarction mortality in the adult health study. Stroke 2004;35:1531-1537.

18. Key TJ, Fraser GE, Thorogood M, et al. Mortality in vegetarians and nonvegetarians: detailed findings from a collaborative analysis of 5 prospective studies. Am J Clin Nutr 1999; 70:516S-524S.

19. Chiu TH, Huang HY, Chen KJ, et al. Relative validity and reproducibility of a quantitative FFQ for assessing nutrient intakes of vegetarians in Taiwan. Public Health Nutr 2014;17:1459-1466.

20. Cheng CL, Kao YH, Lin SJ, Lee CH, Lai ML. Validation of the national health insurance research database with ischemic stroke cases in Taiwan. Pharmacoepidemiol Drug Saf 2011;20:236-242.

21. Korn EL, Graubard BI, Midthune D. Time-to-event analysis of longitudinal follow-up of a survey: choice of the time-scale. Am J Epidemiol 1997;145:72-80.

22. Lamarca R, Alonso J, Gomez G, Munoz A. Left-truncated data with age as time scale: an alternative for survival analysis in the elderly population.J Gerontol A Biol Sci Med Sci 1998;53:M337-M343.

23. Wolbers M, Koller MT, Stel VS, et al. Competing risks analyses: objectives and approaches. Eur Heart J 2014;35:2936-2941.

24. Chuang SY, Chiu TH, Lee CY, et al. Vegetarian diet reduces the risk of hypertension independent of abdominal obesity and inflammation: a prospective study. J Hypertens 2016;34:2164-2171.

25. Chiu THT, Pan WH, Lin MN, Lin CL. Vegetarian diet, change in dietary patterns, and diabetes risk: a prospective study. Nutr Diabetes 2018;8:12.

26. Amarenco P, Labreuche J. Lipid management in the prevention of stroke: review and updated meta-analysis of statins for stroke prevention. Lancet Neurol 2009;8: 453-463.

27. Carlsen $\mathrm{MH}$, Halvorsen $\mathrm{BL}$, Holte $\mathrm{K}$, et al. The total antioxidant content of more than 3100 foods, beverages, spices, herbs and supplements used worldwide. Nutr J 2010;9: 3.

28. Higashi Y, Noma K, Yoshizumi M, Kihara Y. Endothelial function and oxidative stress in cardiovascular diseases. Circ J 2009;73:411-418.

29. Ozawa M, Yoshida D, Hata J, et al. Dietary protein intake and stroke risk in a general Japanese population: the Hisayama Study. Stroke 2017;48:1478-1486.

30. Spence JD. Nutrition in stroke prevention. Semin Neurol 2017;37:259-266. 
31. Threapleton DE, Greenwood DC, Evans CE, et al. Dietary fiber intake and risk of first stroke: a systematic review and meta-analysis. Stroke 2013;44:1360-1368.

32. Tang WH, Wang Z, Levison BS, et al. Intestinal microbial metabolism of phosphatidylcholine and cardiovascular risk. N Engl J Med 2013;368:1575-1584.

33. Zhu W, Gregory JC, Org E, et al. Gut microbial metabolite TMAO enhances platelet hyperreactivity and thrombosis risk. Cell 2016;165:111-124.

34. Nie J, Xie L, Zhao BX, et al. Serum trimethylamine N-oxide concentration is positively associated with first stroke in hypertensive patients. Stroke 2018;49:2021-2028.

35. Wu WK, Chen CC, Liu PY, et al. Identification of TMAO-producer phenotype and host-diet-gut dysbiosis by carnitine challenge test in human and germ-free mice. Gut 2019;68:1439-1449.

36. Tong TYN, Appleby PN, Bradbury KE, et al. Risks of ischaemic heart disease and stroke in meat eaters, fish eaters, and vegetarians over 18 years of follow-up: results from the prospective EPIC-Oxford study. BMJ 2019;366:14897.

37. Ebrahim S, Sung J, Song YM, Ferrer RL, Lawlor DA, Davey Smith G. Serum cholesterol, haemorrhagic stroke, ischaemic stroke, and myocardial infarction: Korean national health system prospective cohort study. BMJ 2006;333:22.

38. Ricci C, Wood A, Muller D, et al. Alcohol intake in relation to non-fatal and fatal coronary heart disease and stroke: EPIC-CVD case-cohort study. BMJ 2018;361: k934.
39. Abebe W. Review of herbal medications with the potential to cause bleeding: dental implications, and risk prediction and prevention avenues. EPMA J 2019;10:51-64.

40. Ndanuko RN, Tapsell LC, Charlton KE, Neale EP, Batterham MJ. Dietary patterns and blood pressure in adults: a systematic review and meta-analysis of randomized controlled trials. Adv Nutr 2016;7:76-89.

41. Larsson SC, Traylor M, Markus HS. Homocysteine and small vessel stroke: a mendelian randomization analysis. Ann Neurol 2019;85:495-501.

42. Iso H, Stampfer MJ, Manson JE, et al. Prospective study of fat and protein intake and risk of intraparenchymal hemorrhage in women. Circulation 2001;103:856-863.

43. Nanri A, Mizoue T, Shimazu T, et al. Dietary patterns and all-cause, cancer, and cardiovascular disease mortality in Japanese men and women: the Japan public health center-based prospective study. PLoS One 2017;12:e0174848.

44. Villegas R, Yang G, Gao YT, et al. Dietary patterns are associated with lower incidence of type 2 diabetes in middle-aged women: the Shanghai Women's Health Study. Int J Epidemiol 2010;39:889-899.

45. B vitamins in patients with recent transient ischaemic attack or stroke in the Vitamins to Prevent Stroke (VITATOPS) trial: a randomised, double-blind, parallel, placebocontrolled trial. Lancet Neurol 2010;9:855-865.

46. Spence JD, Yi Q, Hankey GJ. B vitamins in stroke prevention: time to reconsider. Lancet Neurol 2017;16:750-760. 


\section{Neurology}

\section{Vegetarian diet and incidence of total, ischemic, and hemorrhagic stroke in 2 cohorts in Taiwan}

Tina H.T. Chiu, Huai-Ren Chang, Ling-Yi Wang, et al.

Neurology 2020;94;e1112-e1121 Published Online before print February 26, 2020

DOI 10.1212/WNL.0000000000009093

This information is current as of February 26, 2020

Updated Information \& Services

References

Citations

Subspecialty Collections

Permissions \& Licensing

Reprints including high resolution figures, can be found at: http://n.neurology.org/content/94/11/e1112.full

This article cites 46 articles, 16 of which you can access for free at: http://n.neurology.org/content/94/11/e1112.full\#ref-list-1

This article has been cited by 4 HighWire-hosted articles: http://n.neurology.org/content/94/11/e1112.full\#\#otherarticles

This article, along with others on similar topics, appears in the following collection(s):

All Cerebrovascular disease/Stroke

http://n.neurology.org/cgi/collection/all_cerebrovascular_disease_strok e

Cohort studies

http://n.neurology.org/cgi/collection/cohort_studies

Infarction

http://n.neurology.org/cgi/collection/infarction

Intracerebral hemorrhage

http://n.neurology.org/cgi/collection/intracerebral_hemorrhage

Information about reproducing this article in parts (figures,tables) or in its entirety can be found online at:

http://www.neurology.org/about/about_the_journal\#permissions

Information about ordering reprints can be found online:

http://n.neurology.org/subscribers/advertise

Neurology ${ }^{\circledR}$ is the official journal of the American Academy of Neurology. Published continuously since 1951, it is now a weekly with 48 issues per year. Copyright Copyright ( 2020 The Author(s). Published by Wolters Kluwer Health, Inc. on behalf of the American Academy of Neurology.. All rights reserved. Print ISSN: 0028-3878. Online ISSN: 1526-632X.

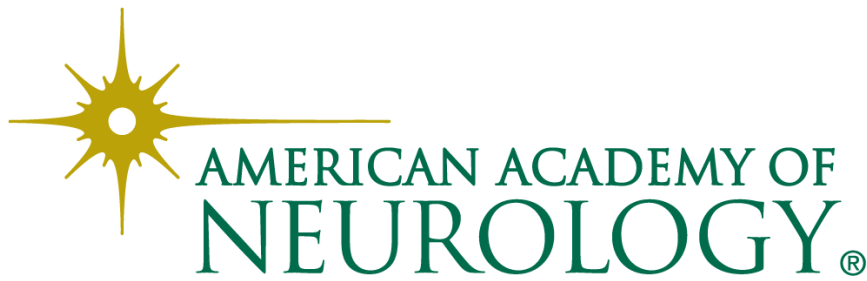

